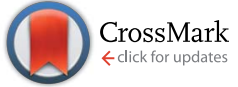

Cite this: RSC Adv., 2017, 7, 13615

Received 5th December 2016 Accepted 22nd February 2017

DOI: $10.1039 / c 6 r a 27768 c$

rsc.li/rsc-advances

\section{Effective thermal transport properties in multiphase biological systems containing carbon nanomaterials}

\author{
Feng Gong, ${ }^{a}$ Jin Liu, ${ }^{b}$ Jian Yang, ${ }^{a}$ Jingang Qin, ${ }^{a}$ Yunlong Yang, ${ }^{a}$ Tingting Feng, ${ }^{a}$ \\ Wenlong Liu, ${ }^{a}$ Hai M. Duong, ${ }^{\star c}$ Dimitrios V. Papavassiliou ${ }^{\star b}$ and Mengqiang $\mathrm{Wu}^{\mathrm{a}}$
}

\begin{abstract}
Here we report computational results from an off-lattice Monte Carlo investigation of the effective thermal transport properties in multiphase biological systems containing carbon nanomaterials. A three-phase system that consists of a cell, healthy tissue and carbon nanotubes (CNTs) was built in silico for this study. The CNTs were embedded in both the cell and the healthy tissue. The effective thermal conductivity $\left(K_{\text {eff }}\right)$ of such biological systems can be predicted by taking into account the dispersion of the CNTs and the interfacial thermal resistances (ITRs) between any pair of components. We quantitatively investigated the effects of the distribution (CNTs at different locations in the system), concentration (0.01-0.1 vol\%), and morphology (diameter of 2-10 nm, length of 200-800 nm) of the CNTs on the $K_{\text {eff }}$ of the biological systems. Additionally, we studied the effects of the ITRs between any pair of components $(0.05-76.5 \times$ $10^{-8} \mathrm{~m}^{2} \mathrm{~K} \mathrm{~W}^{-1}$ ) on the $K_{\text {eff }}$ of the biological systems. The results showed that greater enhancement of the $K_{\text {eff }}$ values of the biological systems can be achieved by using longer CNTs in higher concentration, and reducing the ITRs between the CNTs and their surroundings. Finally, CNTs embedded on the cell membrane have a stronger effect than being dispersed within the cell or in the tissue surrounding the cell.
\end{abstract}

\section{Introduction}

Carbon nanomaterials, especially carbon nanotubes (CNTs) and graphene, have shown superior electrical, mechanical and thermal characteristics, ${ }^{1-3}$ which enable them to be used in diverse applications. For instance, in the biomedicine field, CNTs and graphene have been regarded as promising heating agents in cancer hyperthermia, ${ }^{\mathbf{4 - 1 1}}$ and as efficient cargos for cancer-drug delivery. ${ }^{12-15}$ For cancer hyperthermia, Lin et al. ${ }^{16}$ conducted photothermal ablation of bone metastasis of breast cancer using PEGylated CNTs both in vitro and in vivo. In the in vitro experiments, with a CNT concentration of $100 \mu \mathrm{g} \mathrm{mL}{ }^{-1}$, a temperature increase larger than $30^{\circ} \mathrm{C}$ was obtained in just $4 \mathrm{~min}$. In the in vivo study, with $10 \mu \mathrm{g}$ of CNTs under near-infrared radiation (NIR), a rapid increase in temperature up to $73.4 \pm 11.98{ }^{\circ} \mathrm{C}$ was achieved in the bone metastasis of breast cancer. Yang et al. ${ }^{\mathbf{1 7}}$ investigated the photothermal therapeutic response of cancer cells to graphene oxide (GO). They synthesized aptamer-gold nanoparticle-hybridized GO to deliver heat to human breast cancer cells. The hybridized GO displayed excellent specific targeting capability,

${ }^{a}$ School of Energy Science and Engineering, University of Electronic Science and Technology of China, Chengdu 611731, China

${ }^{b}$ School of Chemical, Biological, and Materials Engineering, University of Oklahoma, Norman, Oklahoma, 73019, USA. E-mail: dvpapava@ou.edu

${ }^{c}$ Department of Mechanical Engineering, National University of Singapore, 117576, Singapore.E-mail: mpedhm@nus.edu.sg anticancer activity, and biocompatibility, suggesting promising breast cancer treatment by using graphene oxide with NIR.

For cancer-drug delivery, Meng et al. ${ }^{12}$ used single-walled CNTs (SWNTs) to deliver doxorubicin to HeLa cancer cells and tumor tissues in chemotherapy. In vitro studies showed that the cut and purified SWNTs (with length less than $500 \mathrm{~nm}$ ) would enter HeLa cells more easily than the long intrinsic SWNTs. In vivo studies showed that SWNTs functionalized with folic acid (FA) could be targeted and localized in the tumor tissue, suggesting an enhanced in vivo behavior of FA-SWNTs. More recently, Wei et $a l .{ }^{13}$ also studied the tumor targeting and drug delivery abilities of graphene oxide. They developed a novel photodynamic therapy (PDT), drug delivery, and phototoxicity on/off nano-system based on graphene oxide (GO). The results showed that the phototoxicity of photosensitizers on the GO could be switched off during the drug delivery course due to the transfer of fluorescence resonance energy. After targeting to the mitochondria, the phototoxicity would be switched on to induce cell death. This work suggested that graphene based biomaterials may not only provide versatile drug carriers, but also enhance the efficiency of PDT.

Considering the composition of the above application systems of carbon nanomaterials, we can find multiple components, such as CNTs/graphene nanosheets, cancer cells, and healthy tissue in the systems. For instance, in the photothermal therapy of cancer using CNTs and NIR, the functionalized CNTs may be located inside, on the cell membrane, or outside of the cancer cell (in the healthy tissue), as illustrated in 


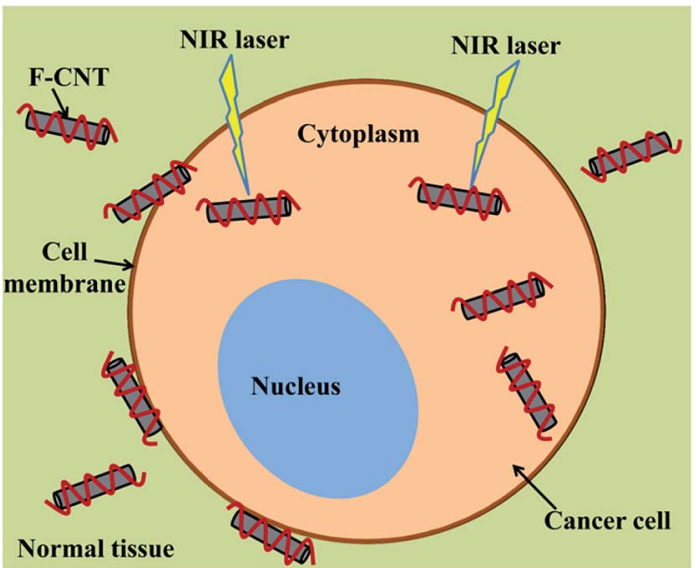

Scheme 1 Schematic plot of the cancer photothermal therapy using functionalized CNTs and near-infrared radiation (NIR) laser. F-CNT represents the functionalized SWNTs.

Scheme 1. As carbon nanomaterials have ultrahigh thermal conductivity $\left(\sim 2000 \mathrm{~W} \mathrm{~m}^{-1} \mathrm{~K}^{-1}\right.$ for CNTs, ${ }^{18} \sim 5000 \mathrm{~W} \mathrm{~m}^{-1} \mathrm{~K}^{-1}$ for graphene ${ }^{19}$ ), they may alter the thermal properties of the multi-phase biological systems. Duong et al. ${ }^{20}$ computationally studied the influence of SWNTs on the thermal transport properties of biological fluids, such as blood. They found that the effective thermal conductivity of two-phase biological systems increased with the concentration of SWNTs, and decreased with the rise of the interfacial thermal resistance between SWNTs and the surrounding biological fluids. However, their study was limited to two-phase systems, which cannot take into account the interactions between different pairs of components in the multiphase biological systems. Therefore, more advanced models are required to quantitatively study the effective thermal properties of multiphase biological systems containing carbon nanomaterials.

In this article, we present a computational off-lattice particlebased approach to model the transfer of heat in a three-phase biological system containing CNTs, cancer cells and healthy tissue. Models containing CNT with different locations (i.e., CNTs in the cancer cell, CNTs on the cancer cell, and CNT out of the cancer cell) were built to represent realistic application systems. The interfacial thermal resistances between any pair of components were quantified through average phonon transmission probabilities, according to the acoustic mismatch theory. The length, diameter and concentration of the CNTs were modified to quantitatively study their effect on the effective thermal conductivity of the three-phase biological system. Such quantitative findings may help researchers to optimize their experimental studies using carbon nanomaterials in biological systems.

\section{Computational methodology}

An off-lattice Monte Carlo approach has been developed to model the transfer of heat in a three-phase biological system. As illustrated in Scheme 1, the three-phase biological system consists of a cancer cell and functionalized SWNTs in the healthy tissue. To mimic a real system, computational models containing SWNTs at different locations were built. As shown in Fig. 1, a cancer cell with a diameter of $1 \mu \mathrm{m}$ was located in the center of a cubic box of normal tissue with a side length of $1.3 \mu \mathrm{m} .{ }^{21} 94$ SWNTs with a diameter of $2 \mathrm{~nm}$ were built inside the cancer cell (Fig. 1a), on the cancer cell membrane (Fig. 1b), or outside of the cancer cell (Fig. 1c). The SWNTs were modeled as solid cylinders with same morphology and thermo-physical properties as the SWNTs, as seen in Table 1. These models can be a representative volume element of realistic systems for the cancer photothermal therapy.

The model in Fig. 1b was chosen as an example to present the off-lattice Monte Carlo methodology. The off-lattice Monte Carlo methodology presented here was applied for studying the thermal transport rather than the mass transport properties. In this study, thermal walkers (hot and cold walkers) were used to quantify the thermal energy. Each walker does not represent water or CNT molecules, but instead, each walker carriers with it an equal amount of heat (as described in ref. 20 and 22). A thermal walker can be present in the water phase or in the CNTs. In each time step, 40000 hot walkers were released from the $y=$ 0 surface and simultaneously, a same number of cold walkers were released from the opposite surface to model the case of constant heat flux through the system, as shown in Fig. 1b. Hot walkers carry same constant positive thermal energy while cold walkers have same negative energy, thus conserving energy in the system. Assuming that the product of the CNT density and heat capacity equals that of the surrounding material (a very reasonable assumption in our case ${ }^{22}$ ), the presence of thermal walkers at a location in our computational box can be directly related to temperature. The temperature profile in the system can be obtained by counting the number of hot and cold walkers in bins that are used to discretize the computational domain, as described in detail as below. Since the hot walkers and cold walkers have same motions, we use thermal walkers to represent both hot and cold walkers.

The thermal walkers are assumed to be shapeless and only have thermal energy, which can be considered as phonons. The collisions between thermal walkers reflect the phonon-phonon scattering, which are complicated and nonlinear interactions. For model simplicity, we ignored the walker collisions in our previous and present work..$^{22,23}$

After the initial release, thermal walkers move randomly with Brownian motion in the healthy tissue. Brownian motion of random walkers can be applied to successfully model the diffusive heat transfer in complex systems. ${ }^{24-26}$ Brownian motion can be also used to model the mass transport of the biological macromolecules (e.g., DNA, protein). ${ }^{27}$ The Brownian motion was described by the position changes in all directions of thermal walkers in each time step. The position changes take values from a normal distribution with a zero mean and a standard deviation, $\sigma_{\mathrm{n}}$, which depends on the thermal diffusivity of the healthy tissue, $D_{\mathrm{n}}$, and is expressed as: ${ }^{28}$

$$
\sigma_{\mathrm{n}}=\sqrt{2 D_{\mathrm{n}} \Delta t}
$$

where $\Delta t$ is the time duration in one time step. Once a thermal walker jumps to the interface between a SWNT and the healthy 
(a)

tissue, the interfacial thermal resistance may hinder the thermal walker from jumping into the SWNT. This interfacial thermal resistance was quantified by a probability, $f_{\mathrm{n} \text {-SwNT }}$, according to the acoustic mismatch theory: ${ }^{29}$

$$
f_{\mathrm{n}-\mathrm{SWNT}}=\frac{4}{\rho_{\mathrm{n}} C_{\mathrm{n}} v_{\mathrm{n}} R_{\mathrm{n}-\mathrm{SWNT}}}
$$

where $\rho_{\mathrm{n}}, C_{\mathrm{n}}, v_{\mathrm{n}}$, and $R_{\mathrm{n}-\mathrm{SwNT}}$ are the density of the healthy tissue, the specific heat capacity of the healthy tissue, the sound velocity in the healthy tissue, and the interfacial thermal resistance between the SWNTs and the healthy tissue, respectively. Thermal walkers may jump into the SWNT with a probability of $f_{\mathrm{n}-\mathrm{SWNT}}$, or stay in the healthy tissue with a probability of $(1-$ $\left.f_{\text {n-SWNT }}\right)$. This is implemented as follows:

(1) The probability, $f_{\text {n-SwNT }}$, is first calculated by using the thermo-physical properties of the healthy tissue $\left(\rho_{\mathrm{n}}, C_{\mathrm{n}}\right.$, and $\left.v_{\mathrm{n}}\right)$ and the interfacial thermal resistances $\left(R_{\mathrm{n}-\mathrm{SWNT}}\right)$.

(2) Since a thermal walker is initially released from the healthy tissue, it jumps once following Brownian motion in each time step. Then our code will check if the new position of the walker is inside any of the SWNTs or not. Every walker in the simulation is tested to see if it can jump inside the SWNTs, and each walker has a probability to enter a SWNT (we do not employ a cut-off distance). However, since the Brownian motion is determined by drawing numbers from a normal distribution, the parameters of this normal distribution determine how large the Brownian jump is. The standard deviation of the Brownian jump is found using Einstein's relation for dispersion, and is related to the thermal diffusivity of the tissue or the cancerous cell (see eqn (1)).

(3) If a walker is in a SWNT, a random number between 0 and 1 will be generated to compare with $f_{\text {n-swNT }}$. If the random number is smaller than or equal to $f_{\mathrm{n} \text {-SwNT }}$, the walker can stay in the SWNT. If the random number is bigger than $f_{\mathrm{n}-\mathrm{SWNT}}$, the walker will move back to the position in the previous step.

(4) If a walker is still in the healthy tissue, no random number is generated and the walker will jump to this new position.

Once a thermal walker jumps into a SWNT, it was assumed to travel in the ballistic transport regime due to the ultrahigh thermal conductivity of SWNTs. ${ }^{30}$ The thermal walkers were distributed uniformly once inside a SWNT. ${ }^{22}$ It has been found that perfect CNTs without defects have ultrahigh thermal conductivity $\left(>2000 \mathrm{~W} \mathrm{~m}^{-1} \mathrm{~K}^{-1}\right)$. $^{31-33}$ Even with defects or functional groups on the surface, CNTs still can have high thermal conductivity up to $\sim 100 \mathrm{~W} \mathrm{~m}^{-1} \mathrm{~K}^{-1}$ or even higher. ${ }^{34-36}$ The thermal conductivity of both perfect CNTs and defected CNTs are much higher (at least 3 orders of magnitude) than those of the tissue and the cancer cell, which are around $0.5 \mathrm{~W}$ $\mathrm{m}^{-1} \mathrm{~K}^{-1} \cdot{ }^{37,38}$ Based on this observation, we assumed ballistic heat transport mechanism in CNTs rather than diffusive heat transfer. In our model, this means an infinite speed of thermal walkers within CNTs. In fact, the infinite speed is relatively infinite, which means that a thermal walker can move everywhere in a single time step when it jumps into a CNT. The length of a CNT can have an effect on whether heat transfer is ballistic or not, in the case when the length of the CNT is comparable or larger than the mean free path of phonons, but this does not occur for the CNTs considered in our work.

When a thermal walker in a SWNT reaches the interface between a SWNT and the healthy tissue, another probability, $f_{\text {SWNT-n }}$, was used to govern whether the walker jumps into the normal tissue or still stays in the SWNT with probability $(1-$ $f_{\text {SWNT-n }}$ ). If a walker jumps out a SWNT, it will first jump to the surface of the SWNT, and then jump to the tissue with once Brownian motion. If a walker still stays in the SWNT, it will be 
Table 1 Physical property parameters used in the simulation

\begin{tabular}{llll}
\hline & Human tissue (biological solution) ${ }^{37}$ & Cancer cell $^{41}$ & SWNT \\
\hline Density $\left(\mathrm{kg} \mathrm{m}^{-3}\right)$ & 1325 & 1025 & $1357(\mathrm{ref} .42)$ \\
Specific heat capacity $\left(\mathrm{J}(\mathrm{kg} \mathrm{K})^{-1}\right)$ & 3750 & 3400 & $841(\text { at } 300 \mathrm{~K})^{43}$ \\
Thermal conductivity $\left(\mathrm{W}(\mathrm{m} \mathrm{K})^{-1}\right)$ & 0.52 & 0.45 & $\sim 3500($ ref. 30$)$ \\
Sound velocity $\left(\mathrm{m} \mathrm{s}^{-1}\right)$ & 1603 & 1500 & $1.29 \times 10^{-7}$ \\
Thermal diffusivity $\left(\mathrm{m}^{2} \mathrm{~s}^{-1}\right)$ & $1.05 \times 10^{-7}$ & $1.03 \times 10^{-3}$ \\
\hline
\end{tabular}

Other simulation parameters

Computational box size (bins)

SWNT diameter (nm)

SWNT length (nm)

SWNTs concentration (\%)

Number of walkers

Simulation time increment (ns)

Interfacial thermal resistance of tissue-SWNT $\left(10^{-8} \mathrm{~m}^{2} \mathrm{~K} \mathrm{~W}^{-1}\right)^{44}$

Interfacial thermal resistance of cell-

SWNT $\left(10^{-8} \mathrm{~m}^{2} \mathrm{~K} \mathrm{~W}^{-1}\right)^{45}$

Interfacial thermal resistance of

tissue-cell $\left(10^{-8} \mathrm{~m}^{2} \mathrm{~K} \mathrm{~W}^{-1}\right)^{46}$

Thermal equilibrium factor
$300 \times 300 \times 300$

$2,6,8,10$

$200,400,600,800$

$0.01,0.02,0.05,0.1$

40000

0.0069

$0.05-50.0$

$0.077-76.5$

$0.10-2.51$

$C_{\mathrm{f}}$ (SWNT-tissue) 0.25
$C_{\mathrm{f}}(\mathrm{SWNT}-\mathrm{cell}) 0.045$ randomly re-distributed in the SWNT. The relation between $f_{\text {SWNT-n }}$ and $f_{\text {n-SWNT }}$ is described below, which is required in order to maintain the second law of thermodynamics in the simulation:

$$
V_{\mathrm{SWNT}} f_{\mathrm{SWNT}-\mathrm{n}}=C_{\mathrm{f}} \sigma_{\mathrm{n}} A_{\mathrm{SWNT}} f_{\mathrm{n}-\mathrm{SWNT}}
$$

where $V_{\mathrm{SWNT}}$ and $A_{\mathrm{SWNT}}$ are the volume and surface area of a SWNT, $\sigma_{\mathrm{n}}$ is the standard deviation of the Brownian motion in the normal tissue, $C_{\mathrm{f}}$ is the thermal equilibrium factor, which depends on the interfacial area and the geometry of SWNTs. ${ }^{39}$ The $C_{\mathrm{f}}$ can be numerically determined at the thermal equilibrium state. ${ }^{21}$ Since SWNTs can lie on the cell membrane (Fig. 1b) or enter the cell (Fig. 1c), thermal walkers in SWNTs may travel into the cancer cell. In the present study, the cell membrane is assumed to have the same thermo-physical properties as the internal of the cell. Due to the similarity between the normal tissue and the cancer cell, thermal walkers in the cancer cell have similar Brownian motion as they jump in the healthy tissue but with a different thermal diffusivity (that of the cancer cell). At the SWNT-cell interface, thermal walkers from either SWNTs or the cancer cell have similar movement to those at SWNT-tissue interface, as described in eqn (2) and (3). At the interface between the normal tissue and the cancer cell, thermal walkers were assumed to have the same probability to cross the interface from both sides. This is because of the relatively large interfacial area between the healthy tissue and the cancer cell. Thermal walkers will be bounced back when they reach the two sides in the $y$ direction, thus maintaining a constant heat flux. Periodic boundary conditions were applied in the $x$ and $z$ directions. All property parameters for the normal tissue, the SWNTs, and the cancer cell are listed in Table 1.

The assumptions in the model are listed as below:
(1) Thermal walkers are characterized as shapeless point particles carrying constant thermal energy.

(2) The interactions between thermal walkers are ignored.

(3) The SWNTs are assumed as solid cylinders with same thermal properties as the SWNTs without considering their functional groups, chirality and defects.

The computational box was divided by 300 mesh points on each side (total $300 \times 300 \times 300$ computational bins) to obtain the temperature profile of the system. The temperature was calculated by counting the number of hot thermal walkers and then subtracting the number of cold walkers. To determine the enhancement in the thermal conductivity of the system by the SWNTs, a reference model with only the normal tissue and the cancer cell was built. With the same heat flux and boundary conditions, the temperature profiles along the $y$ direction in the multiphase systems and the reference model are related as ${ }^{40}$

$$
q^{\prime \prime}=-K_{\text {eff }} \frac{\mathrm{d} T_{\mathrm{S}}}{\mathrm{d} y}=-K_{\mathrm{b}} \frac{\mathrm{d} T_{\mathrm{b}}}{\mathrm{d} y}
$$

where $q^{\prime \prime}, T_{\mathrm{S}}$, and $T_{\mathrm{b}}$ are the applied constant heat flux, the temperature in the system with SWNTs, and the temperature in the reference model without SWNTs. $K_{\text {eff }}$ and $K_{\mathrm{b}}$ are the effective thermal conductivity of the biological systems with SWNTs and the thermal conductivity of the reference model. The enhancement of the thermal conductivity of the system by adding SWNTs can be obtained from eqn (4) and described as

$$
K_{\text {eff }} / K_{\mathrm{b}}=\frac{\mathrm{d} T_{\mathrm{b}}}{\mathrm{d} y} / \frac{\mathrm{d} T_{\mathrm{S}}}{\mathrm{d} y}
$$

Regarding the validation of the model, one can refer to our prior publication (ref. 39). In that work, we presented extensive validation results of the Monte-Carlo model for simulation of 
smaller systems, such as heat transfer from point and line sources of heat, as well as in simple multiphase systems (e.g., a drop suspended inside a different fluid) with CNTs. The model and the movement of each thermal walker were implemented by an in-house algorithm programed with Fortran 95. All the simulations were conducted on super-computers.

\section{Results and discussion}

\subsection{Effect of the SWNT distribution and concentration on the $K_{\text {eff }}$ of the biological system}

We built the models with SWNTs distributed inside, on the cell membrane and outside of the cancer cell to study the effect of SWNT location on the effective thermal conductivity $\left(K_{\text {eff }}\right)$ of the biological system. As presented in Fig. 2, SWNTs with different locations relative to the cancer cell have quite different effects on the $K_{\text {eff }}$ of the biological systems. The CNTs lying on the cancer cell membrane induced the largest enhancement of the $K_{\text {eff }}$, followed by the CNTs located out of the cancer cell, and lastly the CNTs distributed inside the cancer cell. This may be because that the CNTs act as efficient heat transfer channels compared with the normal tissue and the cancer cell. The CNTs lying on the cell membrane can accelerate the heat transferring to the CNTs, and then directly to the cancer cell, which induces a uniform temperature distribution on the system. In the other two systems, the CNTs only exchange heat with one of the other two components (either tissue-CNT-tissue, or cell-CNT-cell). These two heat transfer paths are less effective than the direct path (tissue-CNT-cell) in the system with CNTs on the cell

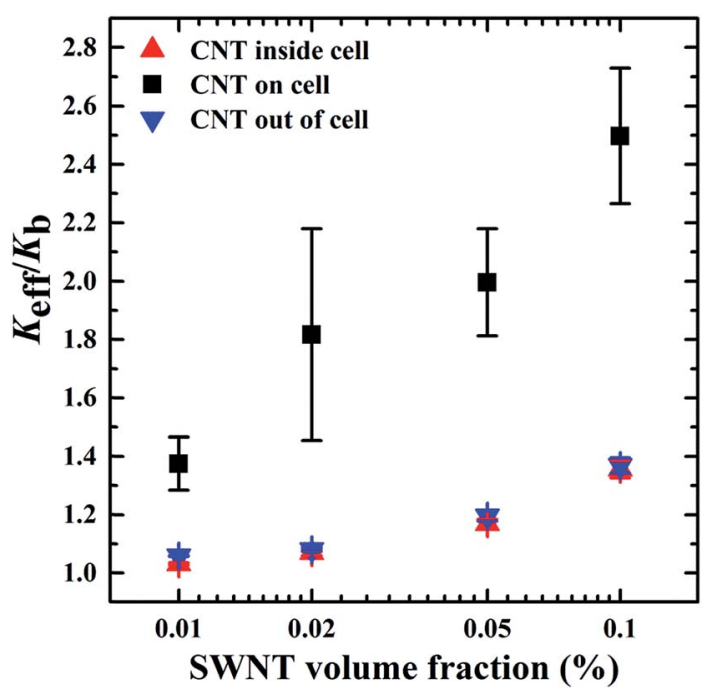

Fig. 2 Effect of the location and concentration of the CNTs on the $K_{\text {eff }}$ of the biological systems. The diameter of the CNTs was $2 \mathrm{~nm}$ and the length of the CNTs was $500 \mathrm{~nm}$. The ITRs at interfaces of tissue-CNT, cell-CNT and tissue-cell were $5.0 \times 10^{-8}, 7.65 \times 10^{-8}$, and $2.51 \times$ $10^{-9} \mathrm{~m}^{2} \mathrm{~K} \mathrm{~W}^{-1}$, respectively. The displayed $K_{\text {eff }}$ in this figure and in the following figures was the average value of the obtained $K_{\text {eff }}$ from 3 separate simulations with different spatial distribution of the SWNTs. The error bars in this figure and the following figures represented the standard deviations of the results obtained from 3 separate simulations with different spatial distribution of the SWNTs. membrane. The CNTs outside of the cancer cell resulted in a slightly higher $K_{\text {eff }}$ than those in the cancer cell. This may be because that the CNTs out of the cancer cell have a more uniform distribution in the whole system than those in the cancer cell. In all three systems, the $K_{\text {eff }}$ increased with increasing CNT concentration. Acting as effective heat transfer channels, more CNTs in the system facilitate the transfer of heat along the heat flux direction, inducing a higher $K_{\text {eff }}$ of the systems.

\subsection{Effect of the interfacial thermal resistance on the $K_{\text {eff }}$ of the biological systems}

The interfacial thermal resistance (ITR) around carbon nanomaterials accounts for the much lower thermal conductivity of the composites than the expected values. ${ }^{47}$ The ITR arises from the difference in the vibrational spectra of the atoms in each component. ${ }^{31}$ The ITRs may influence the transfer of heat in the biological system. ${ }^{21}$ The ITRs were quantified by an average phonon transmission probability according to the acoustic mismatch theory. Fig. 3 is a presentation of the effect of ITR between the normal tissue and the SWNTs $\left(R_{\mathrm{ti}-\mathrm{SWNT}}\right)$ on the $K_{\text {eff }}$ of the biological systems. When the SWNTs are located inside the cancer cell, the SWNTs have no direct contact with the healthy tissue. Therefore, there is no $R_{\mathrm{ti}-\mathrm{SwNT}}$ in the model with the CNTs inside the cancer cell. As shown in Fig. 3, in both models, the $K_{\text {eff }}$ decreases with increasing $R_{\text {ti-swnT }}$. Compared with the healthy tissue, the CNTs with ultrahigh thermal conductivity dominate the transfer of heat in the biological systems. Higher $R_{\mathrm{ti}-\mathrm{SwNT}}$ means that the heat is prevented from

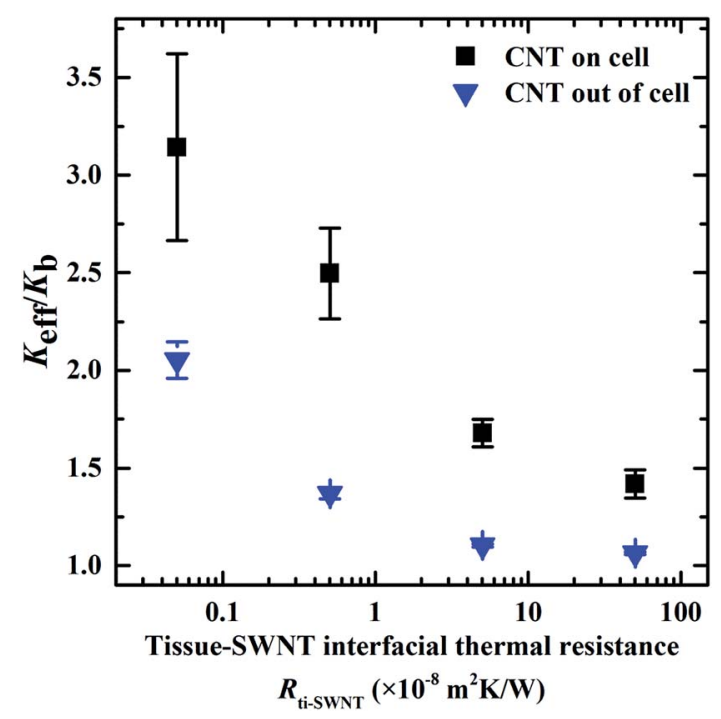

Fig. 3 Effect of the interfacial thermal resistance between the normal tissue and the SWNTs $\left(R_{\mathrm{ti}-S W N T}\right)$ on the $K_{\text {eff }}$ of the biological systems. $R_{\mathrm{ti}-\mathrm{SWNT}}$ was varied in the range of $0.05-50.0 \times 10^{-8} \mathrm{~m}^{2} \mathrm{~K} \mathrm{~W}^{-1,44}$ corresponding to the phonon transmission probability from 0.001 to 1.0. The ITRs at interfaces of cell-CNT and tissue-cell were kept constant as $7.65 \times 10^{-8}$, and $2.51 \times 10^{-9} \mathrm{~m}^{2} \mathrm{~K} \mathrm{~W}^{-1}$. The diameter of the CNTs was $2 \mathrm{~nm}$, the length of the CNTs was $500 \mathrm{~nm}$ and the volume faction of the CNTs was $0.05 \%$. 
transferring in the healthy tissue and rather prefers to be transferred along the CNTs, inducing a lower $K_{\text {eff }}$ of the system.

Similarly, the ITR between the SWNTs and the cancer cell ( $R_{\text {ce-SwnT }}$ ) was changed to study its effect on the $K_{\text {eff }}$ of the biological systems. The $R_{\text {ce-swnt }}$ was modified from $0.077 \times$ $10^{-8} \mathrm{~m}^{2} \mathrm{~K} \mathrm{~W}^{-1}$ to $76.5 \times 10^{-8} \mathrm{~m}^{2} \mathrm{~K} \mathrm{~W}^{-1}$, which corresponds to phonon transmission probability of 0.001-1.0. Similar to the $R_{\mathrm{ti}-\mathrm{SWNT}}$, a larger $R_{\text {ce-SWNT }}$ resulted in a lower $K_{\text {eff }}$ of the systems, as shown in Fig. 4. This mechanism can be explained as in the case of $R_{\mathrm{ti}-\mathrm{SWNT}}$. The SWNTs are the dominant heat transfer channels compared to the cancer cell. Higher $R_{\text {ce-SwnT }}$ prevents the heat transferring from the cancer cell to the SWNTs, lowering the enhancement of the $K_{\text {eff }}$. In the model with the SWNTs located inside the cancer cell, when the $R_{\text {ce-swnT }}$ exceeds $1.0 \times 10^{-7} \mathrm{~m}^{2} \mathrm{~K} \mathrm{~W}^{-1}$, there is almost no improvement in the $K_{\text {eff }}$ of the biological systems. It can be concluded from Fig. 3 and 4 , lower $R_{\text {ce-SWNT }}$ and $R_{\text {ce-SwNT }}$ are preferred to enhance the thermal transport properties in the biological systems. This can be achieved by functionalizing the CNTs with proper surfactants (e.g., DNA, folic acid, and biopolymer) to bridge the atom vibrational spectra in different components. ${ }^{48-50}$

Fig. 5 is a plot of the effect of the ITR between the normal tissue and the cancer cell $\left(R_{\mathrm{ti}-\mathrm{ce}}\right)$ on the $K_{\text {eff }}$ of the biological systems. The $R_{\mathrm{ti}-\mathrm{ce}}$ exists in all three models. Different from the $R_{\mathrm{ti}-\mathrm{SWNT}}$ and the $R_{\text {ce-SwNT }}$, the $R_{\mathrm{ti}-\mathrm{ce}}$ did not show a significant effect on the $K_{\text {eff. }}$ In the models with CNTs located on the cancer cell membrane or outside of the cell, thermal walkers in the normal tissue have higher possibility to jump into the SWNTs rather than into the cancer cell. This is due to the relatively

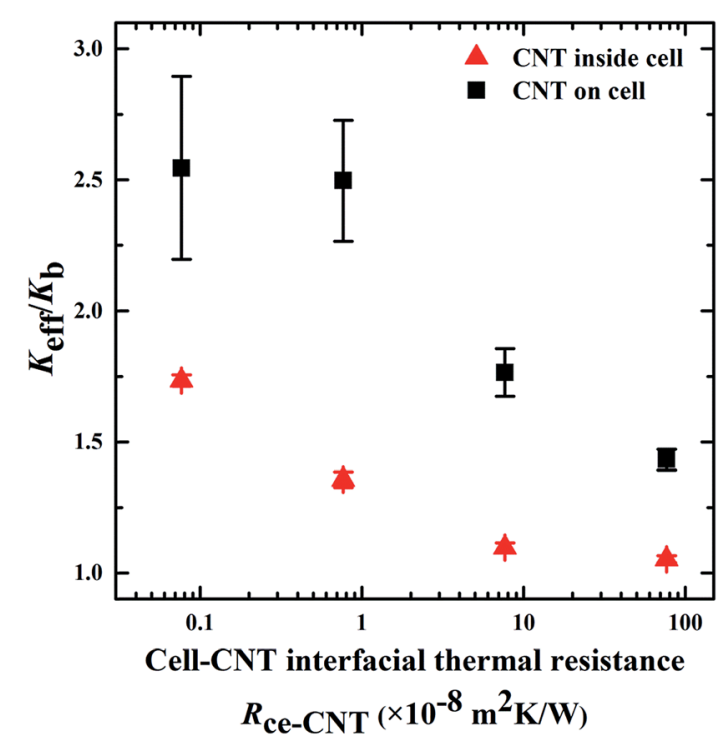

Fig. 4 Effect of the interfacial thermal resistance between the cancer cell and the SWNTs $\left(R_{\text {ce-sWNT }}\right)$ on the $K_{\text {eff }}$ of the biological systems. The $R_{\text {ce-SWNT }}$ was varied in the range of $0.077-76.5 \times 10^{-8} \mathrm{~m}^{2} \mathrm{~K}$ $\mathrm{W}^{-1}, 45$ corresponding to the phonon transmission probability from 0.001 to 1.0. The ITRs at interfaces of tissue-CNT and tissue-cell were kept constant as $5.0 \times 10^{-8}$ and $2.51 \times 10^{-9} \mathrm{~m}^{2} \mathrm{~K} \mathrm{~W}^{-1}$. The diameter of the CNTs was $2 \mathrm{~nm}$, the length of the CNTs was $500 \mathrm{~nm}$ and the volume faction of the CNTs was $0.05 \%$. larger interfacial area between the normal tissue and the SWNTs. This weakens the heat transfer at the interface between the healthy tissue and the cancer cell, thus reducing the effect of the $R_{\mathrm{ti}-\mathrm{ce}}$. However, in the model with CNTs locating inside the cancer cell, the $K_{\text {eff }}$ slightly decreases with increasing $R_{\text {ti-ce }}$. This is because there is no direct contact between the healthy tissue and the SWNTs. Thermal energy will transfer to the cancer cell and then to the SWNTs, which enhances the effect of the $R_{\mathrm{ti}-\mathrm{ce}}$.

\subsection{Effect of the morphology of the SWNTs on the $K_{\text {eff }}$ of the biological systems}

The diameter of the CNTs was modified from 2 to $10 \mathrm{~nm}$ to investigate its effect on the $K_{\text {eff }}$ of the biological systems (Fig. 6). In the models with the CNTs located either inside the cancer cell or outside of the cell, the $K_{\text {eff }}$ slightly decreases with the rise of the CNT diameter. This may be explained as follows: (i) the increase of the CNT diameter facilitates the heat transfer in the radial direction of the CNTs, thus weakening the heat transfer along the heat flux direction; (ii) at the same CNT concentration, the CNTs with increased diameter have decreased interfacial area, reducing the heat exchange between the CNTs and other components. When the CNTs lie on the cancer cell membrane, they obtain a non-uniform distribution in the system. This diminishes the effect of the radial heat transfer and the decreased interfacial area of the CNTs with bigger diameter on the $K_{\text {eff. }}$ Therefore, the CNT diameter did not display an apparent effect on the $K_{\text {eff }}$ when the CNTs located on the cancer cell.

The effect of the CNT length on the $K_{\text {eff }}$ of the biological systems was investigated by varying the CNT length in the range

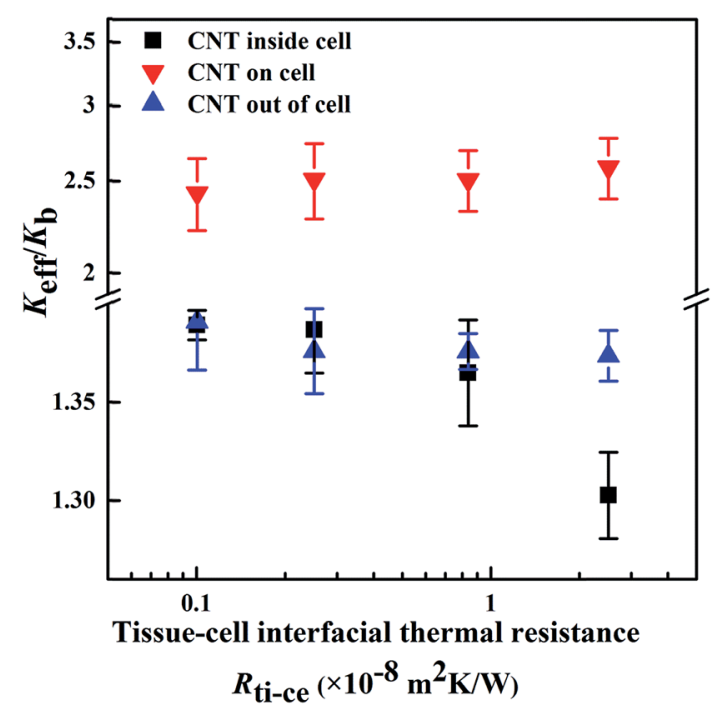

Fig. 5 : Effect of the interfacial thermal resistance between the normal tissue and the cancer cell $\left(R_{\mathrm{ti}-\mathrm{ce}}\right)$ on the $K_{\text {eff }}$ of the biological systems. The $R_{\mathrm{ti}-\mathrm{ce}}$ was varied in the range of $0.10-2.51 \times 10^{-8} \mathrm{~m}^{2} \mathrm{~K} \mathrm{~W}^{-1,46}$ corresponding to the phonon transmission probability from 0.02 to 0.5 . The ITRs at interfaces of tissue-CNT and cell-CNT were $5.0 \times$ $10^{-8}$ and $7.65 \times 10^{-8} \mathrm{~m}^{2} \mathrm{KW}^{-1}$. The diameter of the CNTs was $2 \mathrm{~nm}$, the length of the CNTs was $500 \mathrm{~nm}$ and the volume faction of the CNTs was $0.05 \%$. 


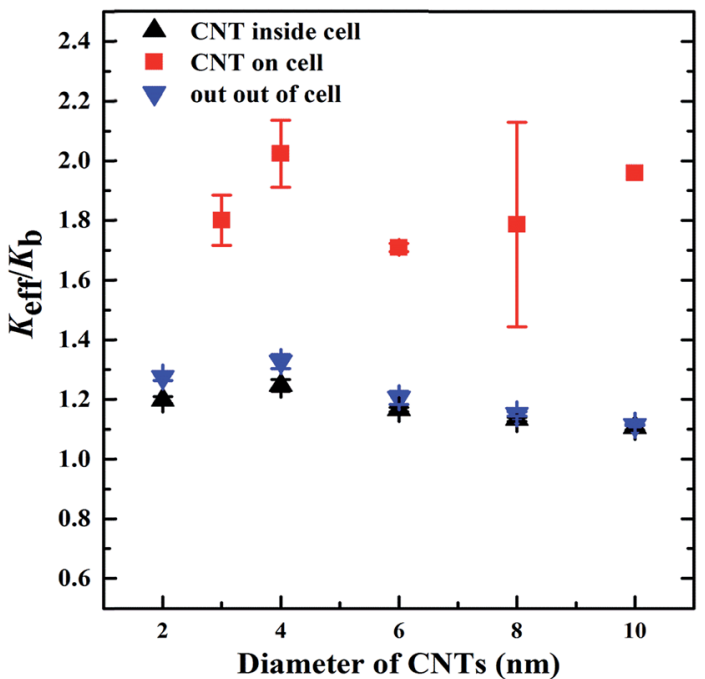

Fig. 6 Effect of the CNT diameter on the $K_{\text {eff }}$ of the biological systems. The diameter was varied from 2 to $10 \mathrm{~nm}$, and the length was kept constant as $500 \mathrm{~nm}$. The ITRs at interfaces of tissue-CNT, cell-CNT and tissue-cell were $5.0 \times 10^{-8}, 7.65 \times 10^{-8}$, and $2.51 \times 10^{-9} \mathrm{~m}^{2} \mathrm{~K}$ $\mathrm{W}^{-1}$, respectively.

of 200-800 nm, while the CNT diameter was kept constant as $2 \mathrm{~nm}$. In all three models, the $K_{\text {eff }}$ greatly increased with the rise of the CNT length (Fig. 7). Longer CNTs transport heat through the system more effectively than the shorter ones, thereby inducing higher $K_{\text {eff }}$ of the biological systems. ${ }^{51,52}$ On the other hand, the reduced stiffness of the longer CNTs may generate phonons with lower vibrational frequency, thus increasing the overlap in the vibrational spectra of the CNTs and other components. $^{23,53}$ The increased overlap in vibrational spectra will lower the interfacial thermal resistance between the CNTs

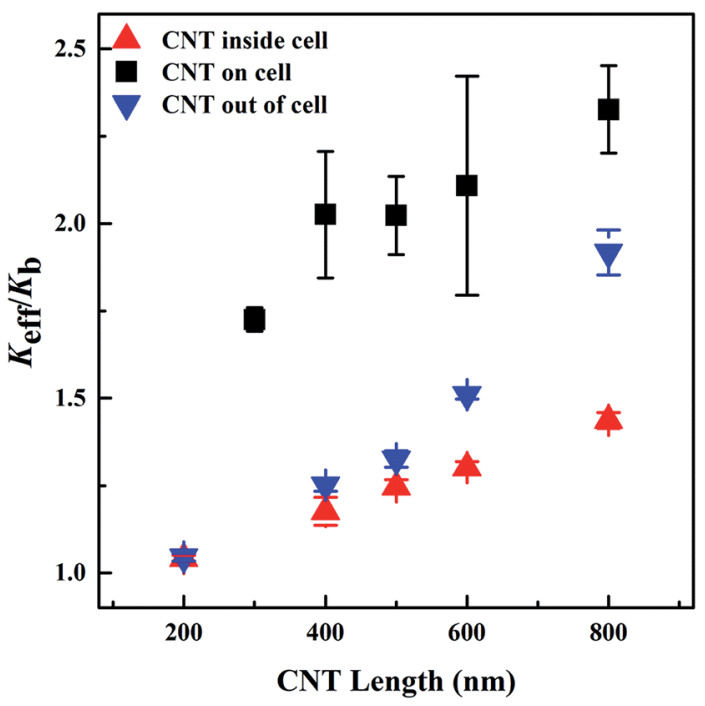

Fig. 7 Effect of the CNT length on the $K_{\text {eff }}$ of the biological systems The length of the CNTs was varied from 200 to $800 \mathrm{~nm}$, and the diameter was kept as $2 \mathrm{~nm}$. The ITRs at interfaces of tissue-CNT, cellCNT and tissue-cell were $5.0 \times 10^{-8}, 7.65 \times 10^{-8}$, and $2.51 \times 10^{-9} \mathrm{~m}^{2}$ $\mathrm{KW}^{-1}$, respectively. and their surroundings, which may significantly enhance the effective thermal transport properties in the biological systems, as discussed in Section 3.2. Compared with the other two systems, the CNT length showed less influence on the $K_{\text {eff }}$ when the CNTs were located inside the cancer cell. This may be due to the aggregated distribution of the CNTs inside the cancer cell. ${ }^{54}$

\section{Conclusion}

In summary, we have quantitatively investigated the effective thermal transport properties of multiphase biological systems containing CNTs via an off-lattice Monte Carlo approach. The quantitative findings show that the CNTs and the ITRs between the CNTs and their surroundings dominate the transfer of heat in the biological systems. The CNTs located on the cancer cell membrane that had higher concentrations and longer lengths would induce higher $K_{\text {eff }}$ of the biological systems. This is due to the ultrahigh thermal conductivity and the increased uniform distribution of the CNTs. The presence of CNTs inside the cancerous cell had the least effect among the different cases studied. The results obtained here indicate that the thermal conductivity of the system can be increased by up to $250 \%$ when the CNTs are on the cell membrane, while it increases by about $20 \%$ when the cells contain the CNTs. The thermal transport properties in the biological systems also can be greatly enhanced by reducing the interfacial thermal resistances between the CNTs and other components, which may be achieved by the proper functionalization of the CNTs. These findings may help researchers to optimize the thermal properties of the biological systems. For example, techniques that functionalize the CNTs with biologically active chemicals, so that they travel through the body to find and deposit on specific cell sites that express proteins when they are cancerous, ${ }^{55-57}$ could have special importance for phototherapy options. When the CNTs attach to the membrane of the cancer cell after proper functionalization, an enhanced $K_{\text {eff }}$ of the biological system can be achieved. The enhanced $K_{\text {eff }}$ can lead to thermal energy transferred more efficiently and faster to the cancerous cells, inducing a more efficient treatment. Our approach can be also modified to investigate the thermal transport properties of other multiphase biological systems containing, for example, graphene nanosheets or other carbon nanomaterials.

\section{Acknowledgements}

We would like to appreciate the financial support from National Natural Science Foundation of China (51602038). Hai M. Duong would like to thank the support from Ministry of Education (MOE) of Singapore (R-397-000-226-112).

\section{Reference}

1 K. S. Novoselov, V. I. Falko, L. Colombo, P. R. Gellert, M. G. Schwab and K. Kim, Nature, 2012, 490, 192-200.

2 Q. Zhang, J.-Q. Huang, W.-Z. Qian, Y.-Y. Zhang and F. Wei, Small, 2013, 9, 1237-1265. 
3 Y. Fang, Y. Lv, F. Gong, Z. Wu, X. Li, H. Zhu, L. Zhou, C. Yao, F. Zhang, G. Zheng and D. Zhao, J. Am. Chem. Soc., 2015, 137, 2808-2811.

4 R. Singh and S. V. Torti, Adv. Drug Delivery Rev., 2013, 65, 2045-2060.

5 M. Orecchioni, R. Cabizza, A. Bianco and L. G. Delogu, Theranostics, 2015, 5, 710-723.

6 S. H. Kim, J. E. Lee, S. M. Sharker, J. H. Jeong, I. In and S. Y. Park, Biomacromolecules, 2015, 16, 3519-3529.

7 K. Cherukula, K. Manickavasagam Lekshmi, S. Uthaman, K. Cho, C.-S. Cho and I.-K. Park, Nanomaterials, 2016, 6, 76. 8 D. K. Chatterjee, P. Diagaradjane and S. Krishnan, Ther. Delivery, 2011, 2, 1001-1014.

9 B. N. Eldridge, B. W. Bernish, C. D. Fahrenholtz and R. Singh, ACS Biomater. Sci. Eng., 2016, 2, 963-976.

10 T. Mocan, C. T. Matea, I. Cojocaru, I. Ilie, F. A. Tabaran, F. Zaharie, C. Iancu, D. Bartos and L. Mocan, Cancer J., 2014, 5, 679-688.

11 O. Akhavan, E. Ghaderi, S. Aghayee, Y. Fereydooni and A. Talebi, J. Mater. Chem., 2012, 22, 13773-13781.

12 L. Meng, X. Zhang, Q. Lu, Z. Fei and P. J. Dyson, Biomaterials, 2012, 33, 1689-1698.

13 Y. Wei, F. Zhou, D. Zhang, Q. Chen and D. Xing, Nanoscale, 2016, 8, 3530-3538.

14 S. Goenka, V. Sant and S. Sant, J. Controlled Release, 2014, 173, 75-88.

15 K. Yang, L. Feng and Z. Liu, Adv. Drug Delivery Rev., 2016, 105, 228-241.

16 Z. Lin, Y. Liu, X. Ma, S. Hu, J. Zhang, Q. Wu, W. Ye, S. Zhu, D. Yang, D. Qu and J. Jiang, Sci. Rep., 2015, 5, 11709.

17 L. Yang, Y.-T. Tseng, G. Suo, L. Chen, J. Yu, W.-J. Chiu, C.-C. Huang and C.-H. Lin, ACS Appl. Mater. Interfaces, 2015, 7, 5097-5106.

18 E. Pop, D. Mann, Q. Wang, K. E. Goodson and H. J. Dai, Nano Lett., 2006, 6, 96-100.

19 A. A. Balandin, S. Ghosh, W. Bao, I. Calizo, D. Teweldebrhan, F. Miao and C. N. Lau, Nano Lett., 2008, 8, 902-907.

20 H. M. Duong, D. V. Papavassiliou, K. J. Mullen, B. L. Wardle and S. Maruyama, Int. J. Heat Mass Transfer, 2009, 52, 55915597.

21 F. Gong, Z. Hongyan, D. V. Papavassiliou, K. Bui, C. Lim and H. M. Duong, Nanotechnology, 2014, 25, 205101.

22 H. M. Duong, D. V. Papavassiliou, L. L. Lee and K. J. Mullen, Appl. Phys. Lett., 2005, 87, 013101.

23 F. Gong, K. Bui, D. V. Papavassiliou and H. M. Duong, Carbon, 2014, 78, 305-316.

24 M. M. Tomadakis and S. V. Sotirchos, J. Chem. Phys., 1993, 98, 616-626.

25 D. V. Papavassiliou, Int. J. Heat Mass Transfer, 2002, 45, 35713583.

26 B. M. Mitrovic, P. M. Le and D. V. Papavassiliou, Chem. Eng. Sci., 2004, 59, 543-555.

27 M. H. Peters, J. Chem. Phys., 2011, 134, 025105.

28 A. Einstein, Ann. Phys., 1905, 17, 891-921.

29 E. T. Swartz and R. O. Pohl, Rev. Mod. Phys., 1989, 61, 605668.

30 A. A. Balandin, Nat. Mater., 2011, 10, 569-581.
31 Z. D. Han and A. Fina, Prog. Polym. Sci., 2011, 36, 914-944.

32 R. H. Baughman, A. A. Zakhidov and W. A. de Heer, Science, 2002, 297, 787-792.

33 M. F. L. De Volder, S. H. Tawfick, R. H. Baughman and A. J. Hart, Science, 2013, 339, 535-539.

34 Q. Zhou, F. Meng, Z. Liu and S. Shi, J. Nanomater., 2013, 2013, 7 .

35 F. Z. Kai Tang, Y. Chen, Y. Li, H. Liao, X. Chen and S. Liu, 2013 IEEE 15th Electronics Packaging Technology Conference, 2013, pp. 817-820.

36 J. Park, M. F. P. Bifano and V. Prakash, J. Appl. Phys., 2013, 113, 034312.

37 C. Sturesson and S. Andersson Engels, Phys. Med. Biol., 1995, 40, 2037-2052.

38 X. Xu, A. Meade and Y. Bayazitoglu, Lasers Med. Sci., 2011, 26, 213-222.

39 F. Gong, D. V. Papavassiliou and H. M. Duong, Numer. Heat Transfer, Part A, 2014, 65, 1023-1043.

40 R. Byron Bird, W. E. Stewart and E. N. Lightfoot, Transport Phenomena, John Wiley \& Sons, 2nd edn, 2007.

41 X. A. Xu, A. Meade and Y. Bayazitoglu, Lasers Med. Sci., 2011, 26, 213-222.

42 G. H. Gao, T. Cagin and W. A. Goddard, Nanotechnology, 1998, 9, 184-191.

43 Y. Xiao, X. H. Yan, J. X. Cao, J. W. Ding, Y. L. Mao and J. Xiang, Phys. Rev. B: Condens. Matter Mater. Phys., 2004, 69, 205415.

44 S. T. Huxtable, D. G. Cahill, S. Shenogin, L. P. Xue, R. Ozisik, P. Barone, M. Usrey, M. S. Strano, G. Siddons, M. Shim and P. Keblinski, Nat. Mater., 2003, 2, 731-734.

45 S. Maruyama, Y. Igarashi, Y. Taniguchi and J. Shiomi, J. Therm. Sci. Technol., 2006, 1, 138-148.

46 H. A. Patel, S. Garde and P. Keblinski, Nano Lett., 2005, 5, 2225-2231.

47 M. Naffakh, A. M. Diez-Pascual, C. Marco and G. Ellis, J. Mater. Chem., 2012, 22, 1418-1425.

48 T. C. Clancy and T. S. Gates, Polymer, 2006, 47, 5990-5996.

49 V. Varshney, S. S. Patnaik, A. K. Roy and B. L. Farmer, J. Phys. Chem. C, 2010, 114, 16223-16228.

50 N. W. S. Kam, M. O'Connell, J. A. Wisdom and H. J. Dai, Proc. Natl. Acad. Sci. U. S. A., 2005, 102, 11600-11605.

51 R. S. Kapadia, B. M. Louie and P. R. Bandaru, J. Heat Transfer, 2014, 136, 011303.

52 A. S. Cherkasova and J. W. Shan, J. Heat Transfer, 2010, 132, 082402.

53 H. Zhong and J. R. Lukes, Phys. Rev. B: Condens. Matter Mater. Phys., 2006, 74, 125403.

54 F. Gong, H. M. Duong and D. V. Papavassiliou, J. Phys. Chem. C, 2015, 119, 7614-7620.

55 F. F. N. Luís, J. K. John, D. V. R. Brent, R. Rajagopal, E. R. Daniel and G. H. Roger, Nanotechnology, 2013, 24, 375104.

56 R. P. Naveen, E. M. David, F. F. N. Luis, T. Yongqiang, E. R. Daniel and G. H. Roger, Nanotechnology, 2007, 18, 235601.

57 M. P. Whitney, D. V. R. Brent, E. R. Daniel and G. H. Roger, Nanotechnology, 2011, 22, 455101. 